\title{
Risk assessment of and targeted intervention for falls in the elderly based on the relationship between physical function levels and risk factors for falls
}

\author{
Susumu Sato ${ }^{1 \#}$, Shinichi Demura ${ }^{2}$, Masanobu Uchiyama $^{3}$ \\ ${ }^{1}$ Kanazawa Institute of Technology, Ishikawa, Japan; ${ }^{\#}$ Corresponding Author: sssato@neptune.kanazawa-it.ac.jp \\ ${ }^{2}$ Graduate School of Natural Science and Technology, Kanazawa University, Ishikawa, Japan \\ ${ }^{3}$ Akita Prefectural University, Akita, Japan
}

Received 22 April 2013; revised 22 May 2013; accepted 2 June 2013

Copyright (C) 2013 Susumu Sato et al. This is an open access article distributed under the Creative Commons Attribution License, which permits unrestricted use, distribution, and reproduction in any medium, provided the original work is properly cited.

\begin{abstract}
Although fall experience is an important predictor of future falls, clinicians place too much emphasis on this factor during risk assessment of falls. This study aimed to determine individuals with high and low fall risk on the basis of the relationship between physical function levels [competence in performing activities of daily living (ADL)] and risk factors for falls (fall experience, locomotive organ impairment, and potential for falling). Participants were 1057 independent community-dwelling elderly individuals. ADL competence and the status of risk factors for falls were evaluated using a questionnaire and the relationship between the two was determined. We calculated the cumulative relative frequency curves for ADL scores after categorizing the participants into several groups according to combinations of risk factors and analyzed those combinations that showed wide individual differences in the cumulative relative frequency curves. Intergroup differences when the participants were categorized on the basis of fall experience alone were small; however, those when the participants were categorized on the basis of locomotive impairment and potential for falling were much greater. On classifying the participants into eight groups on the basis of locomotive organ health, fall risk, and fall experience, we found that fallers with locomotive impairment and a high fall risk exhibited the lowest ADL scores while fallers without locomotive impairment and with a low fall risk exhibited the highest ADL scores. Fall risk assessments that are
\end{abstract}

\footnotetext{
"Competing interest: We declare that we have no competing interests.
}

overtly dependent on fall experience are fundamentally limited. Grouping key risk factors can help in determining target groups, including highrisk fallers, low-risk fallers, and high-risk nonfallers, which require primary preventive measures among the community-dwelling elderly.

Keywords: Locomotive Organ Impairment; Fall Experience; ADL Competence

\section{INTRODUCTION}

Japan has the highest longevity indices in the world (WHO), with a healthy life expectancy of 73 years for men and 78 years for women [1,2]. Healthy aging by bridging the gap between life expectancy and healthy life expectancy is therefore important for both individuals and society. A decline in physical function and locomotive impairment decrease healthy life expectancy, and falling is a typical event that can induce such sequelae among the elderly by causing fractures and enhancing inactivity [3-5]. In addition, a fall experience increases the risk of future falls. Several interventions that aim to prevent future falls or maintain or improve physical function have been introduced to minimize the fall risk and other sequelae.

Independent community-dwelling elderly individuals include those with good physical health and outstanding physical function as well as those with impaired locomotion, decreased physical function, and low levels of activity. Although previous studies have emphasized on fall experience as a risk factor during fall risk assessment [6], the diversity in physical function and fall risk levels among the community-dwelling elderly necessitate the need for analysis of additional factors in order to devise appropriate preventive measures against falls. For exam- 
ple, it may be inappropriate to uniformly categorize all elderly individuals as high-risk fallers following a fall experience, considering that even young individuals experience falls. Therefore, there is a need to discriminate between high-risk fallers and low-risk fallers and determine individualized interventions. In addition, high-risk nonfallers also need to be identified as a target group for primary fall prevention.

Assessing the health of locomotive organs and risk factors for falls is reportedly essential to apply effective primary preventive measures for the community-dwelling elderly $[7,8]$. Fall experiences together with the number of falls are useful predictors of future falls [9]. Previous studies developed procedures to screen for elderly individuals at a high risk of falling using fall experience as a validity criterion and examination of physical functional characteristics. However, assessments that are overtly dependent on fall experiences may miss out on some important elderly groups that require targeted intervention. The Japanese Orthopaedic Association has proposed the concept of a "locomotive syndrome," which is an umbrella term referring to locomotive conditions requiring care services or high-risk locomotive conditions that may soon require care services because of impairments in locomotive organs $[5,10]$. Locomotive diseases are ranked as major reasons attributing to the requirement of long-term care services among the independent Japanese elderly and cannot be overlooked while determining appropriate primary preventive measures against falls [11]. Although locomotive organ health and fall risk factors are often assessed independently, combining these assessments may provide useful information for determining the required preventive measures and subsequent interventions.

While independent community-dwelling elderly individuals cannot be referred to as extremely healthy individuals, many of them have no serious disorders or impairments. The low-risk individuals in this population may be defined as those with high levels of physical function and activity, healthy locomotive organs, and a low potential for falling. In contrast, high-risk individuals can be defined as those with low levels of physical function and activity, unhealthy locomotive organs, and a high potential for falling. However, fall risk assessment based on the classification of community-dwelling elderly individuals as high-risk fallers, low-risk fallers, lowrisk nonfallers, and high-risk nonfallers has not been performed in previous studies. The characteristics of each category of fallers also remain to be clarified.

The aims of this study were as follows: to deter-mine the relationship between physical function [activities of Daily Living (ADL) competence] and the status of risk factors for falls, including fall experience, locomotive organ health, and fall risk level; to examine whether a target group requiring primary preventive measures against falls, including high-risk fallers and high-risk nonfallers, could be identified on the basis of this relationship; and to confirm whether fall experience could independently identify high-risk fallers among independent elderly community-dwelling individuals.

\section{METHODS}

\subsection{Participants}

A total of 1530 independent community-dwelling elderly individuals were recruited from Ishikawa, Fukui, Aichi, and Oita Prefectures in Japan. A questionnaire that included questions related to fall experience, locomotive organ impairment, fall risk, and ADL competence was then administered to all participants. The response rate was 85\% (1296/1530). Participants who provided incomplete responses were excluded, and 1057 respondents (69\%) were eventually subjected to statistical analysis. A written explanation of the purpose of this study was provided and written informed consent was obtained from each participant. This study was approved by the Ethics Committee on Human Experimentation of Faculty of Human Science, Kanazawa University.

\subsection{Fall Risk Assessment}

Fall risk was assessed using Demura's fall risk assessment chart (DFRA), which includes 50 fall risk assessment items representing five key risk factors: potential for falling, physical function, disease and physical symptom, environment, and "behavior and character" [12]. All responses were recorded on a dichotomous scale (yes or no), with one point being assigned for each response falling into the high-risk category. The risk factor score was calculated by adding individual scores of the structural items of each risk factor. In the DFRA, a comprehensive fall risk level is evaluated using the potential for falling score. Potential for falling is a factor concerned with the presence of fall precursors and is assessed by the following three questions: "Have you often stumbled?", "In the past year, have you felt like you might fall down?”, and "Have you ever been told that you look like you might fall down?" The cutoff point is set at a single point (i.e. the screening assessment) $[13,14]$. Participants were judged to be high-risk individuals or low-risk individuals on the basis of their potential for falling score.

\subsection{Assessment of Fall Experience}

Participants were asked to rate their experiences of falling in the previous 12 months, and they were classified as fallers and nonfallers on the basis of their response to this question. 


\subsection{Assessment of Locomotive Organ Impairment}

Participants were questioned whether they had any joint or musculoskeletal disorders (pain, complaints, diseases, disabilities) in the locomotive organs (feet, ankle, knees, hips) and trunk and were instructed to answer as yes or no for each site. The participants self assessed the status of their locomotive organ health. This study identified an individual to have locomotive impairments if he/ she was symptomatic or had a disorder involving any of the abovementioned sites.

\subsection{Assessment of ADL Competence}

To evaluate physical function, the ADL assessment sheet approved by the Ministry of Education, Culture, Sports, Science and Technology in Japan was used [15]. This ADL assessment sheet comprises 12 items representing four domains: locomotive activities, manual activities, balancing activities, and posture-changing activities. Each item is graded on a 3-point scale and scores are combined to achieve a total score (minimum 12, maximum 36) and subscale scores. Higher scores are associated with better physical function.

\subsection{Statistical Analyses}

This study classified the participants into several groups on the basis of risk factor assessment: fall experience (faller and nonfaller), locomotive organs impairment, and potential for falling (high-risk individual and low-risk individual). ADL competence was compared among these groups to examine the relationship between physical function and risk factors. Statistical differences in mean ADL scores among these groups were confirmed by ANCOVA (adjusted by age effect).

To compare the distribution characteristics of ADL scores among groups, a frequency distribution was converted to a cumulative relative frequency distribution curve for each group. Among the groups with different physical function levels based on ADL competence, their cumulative relative frequency distribution curves were positioned to either side. For example, the distribution curve of a group with high ADL competence tends to position on the right side (high-score area), whereas that of a low ADL competence group tends to position on the left side (low score area). We considered that group differences in distribution curves would change depending on specific fall risk factors and their combinations used for grouping and that individual factors or combinations that showed greater intergroup differences could provide useful information for determining primary preventive measures against falls.

Participants in this study were grouped as described below.

a) Classification by fall experience: On the basis of fall experience in the previous 12 months, participants were divided into fallers and nonfallers.

b) Classification by locomotive organ impairment and potential for falling: On the basis of locomotive organ impairment and potential for falling, participants were divided into four groups: a no locomotive impairment and low fall risk group (NLLR), a no locomotive impairment and high fall risk group (NLHR), a locomotive impairment and low fall risk group (LLR), and a locomotive impairment and high fall risk group (LHR).

c) Classification by fall experience, locomotive organ impairment, and potential for falling: On the basis of fall experience, locomotive organ impairment, and potential for falling, participants were divided into eight groups: nonfallers with no locomotive impairment and low fall risk (NLLR-nonfaller), nonfallers with no locomotive impairment and high fall risk (NLHR-nonfaller), nonfallers with locomotive impairment and low fall risk (LLR-nonfaller), nonfallers with locomotive impairment and high fall risk (LHR-nonfaller), fallers with no locomotive impairment and low fall risk (NLLR-faller), fallers with no locomotive impairment and high fall risk (NLHR-faller), fallers with locomotive impairment and low fall risk (LLR-faller), and fallers with locomotive impairment and high fall risk (LHR-faller).

For all three categories mentioned above, cumulative relative frequency distribution curves were calculated for each group and statistical differences in mean ADL scores among groups were determined.

\section{RESULTS}

\subsection{Classification by Fall Experience}

The mean ADL score was significantly higher for the nonfallers than for the fallers (Table 1). In addition, the cumulative relative frequency distribution curve for the nonfallers was positioned more toward the right (high score area) compared with that for the faller group (Figure 1).

\subsection{Classification by Locomotive Organ Impairment and Potential for Falling}

Table 2 and Figure 2 show the results for the NLLR,

Table 1. The results for the fallers and nonfallers.

\begin{tabular}{ccccccc}
\hline & \multicolumn{2}{c}{ Nonfaller } & \multicolumn{2}{c}{ Faller } & \multicolumn{2}{c}{ ANCOVA } \\
\cline { 2 - 7 } & Mean & SD & Mean & SD & F & p \\
\hline ADL score & 27.6 & 5.3 & 25.1 & 6.4 & 25.5 & 0.0 \\
Mean age $^{\#}$ & 72.2 & 6.9 & 74.0 & 7.6 & 412.1 & 0.0 \\
\hline
\end{tabular}

${ }^{\#}$ Covariate. 


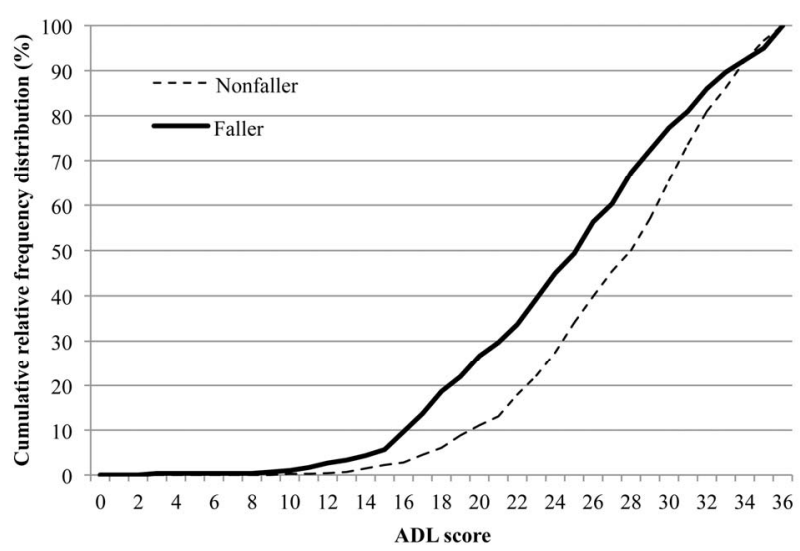

Figure 1. The cumulative relative frequency distribution curve for the faller and nonfaller.

NLHR, LLR, and LHR groups. The mean ADL score was significantly different among groups, with the NLLR group exhibiting the highest score, followed by the NLHR, LLR, and LHR groups. Cumulative relative frequency distribution curves for the two groups without locomotive impairment (NLLR and NLHR) were positioned more toward the right (high score area) compared with that for the two groups with locomotive impairment (LLR and LHR). Among groups with the same locomotive condition, the distribution curves for the high fall risk groups tended to be positioned more toward the left (low score area). The influence on the distribution of ADL score tended to be greater with locomotive impairment than with fall risk level. Furthermore, intergroup differences in the distribution characteristics of ADL scores tended to be greater when participants were classified according to locomotive impairment and potential for falling than when they were classified according to fall experience.

\subsection{Classification by Fall Experience, Locomotive Impairment, and Potential for Falling}

Table 3 and Figure 3 show the results for the NLLRnonfaller, NLHR-nonfaller, LLR-nonfaller, LHR-nonfaller, NLLR-faller, NLHR-faller, LLR-faller, and LHRfaller groups. The mean ADL score was significantly different among groups, with the LHR-faller group exhibit- ing the lowest score. The two groups without locomotive impairment and with a low fall risk (NLLR-nonnfaller and NLLR-faller) exhibited high ADL scores, albeit with no significant differences. As shown in Figure 3, the distribution curve for the NLLR-faller group was positioned more toward the right side (high score area) compared with that for the NLLR-nonfaller group. This suggests that fallers include elderly individuals with high physical function. Furthermore, there were no significant differences in mean ADL scores between pairs of groups with the same locomotive conditions and fall risk (NLLRfaller and nonfaller, NLHR-faller and nonfaller, LLRfaller and nonfaller, and LHR-faller and nonfaller).

\section{DISCUSSION}

In Japan, fall prevention in the elderly is becoming an issue of increasing importance, particularly with regard to primary preventive measures against falls in the community-dwelling elderly. Independent elderly community-dwelling individuals are a heterogeneous group with a wide range of physical functions and independence levels, ranging from those who are physically very fit and active to those who have minimal physical function. Improvement of physical function is an essential goal of primary preventive measures against falls. Individuals with fall experience reportedly have a high re-fall rate; therefore, fall experience is considered to be an important factor in fall risk assessment [3,9]. However, because of the diversity in physical function and independence levels in the independent community-dwelling elderly, prediction of future falls on the basis of fall experience alone may not be entirely reliable. Not all fallers are at risk of falling in the future, and not all nonfallers are at negligible risk of falling in the future. Classification of elderly individuals with good physical function as high-risk fallers on the basis of a single fall experience may introduce inactivity into their daily life and increase the fall risk as a consequence. Further, a wrong estimation of high-risk and low-risk individuals can lead to a misrepresentation of the magnitude of the problem and the inappropriate management of high-risk individuals. It is particularly important to distinguish high-risk fallers from low-risk faller among the community-dwe-

Table 2. The results for classification by locomotive organ impairment and potential for falling.

\begin{tabular}{|c|c|c|c|c|c|c|c|c|c|c|c|}
\hline & \multicolumn{2}{|c|}{$\begin{array}{c}\text { NLLR } \\
(\mathrm{n}=415)\end{array}$} & \multicolumn{2}{|c|}{$\begin{array}{c}\text { NLHR } \\
(n=88)\end{array}$} & \multicolumn{2}{|c|}{$\begin{array}{c}\text { LLR } \\
(\mathrm{n}=373)\end{array}$} & \multicolumn{2}{|c|}{$\begin{array}{c}\text { LHR } \\
(\mathrm{n}=181)\end{array}$} & \multicolumn{3}{|r|}{ ANCOVA } \\
\hline & Mean & $\mathrm{SD}$ & Mean & $\mathrm{SD}$ & Mean & $\mathrm{SD}$ & Mean & SD & $\mathrm{F}$ & $\mathrm{p}$ & Multiple comparison \\
\hline Mean age & 70.7 & 6.7 & 71.6 & 7.4 & 73.8 & 6.9 & 75.5 & 7.1 & 333.1 & 0.0 & \\
\hline
\end{tabular}

NLLR: a no locomotive impairment and low fall risk group; NLHR: a no locomotive impairment and high fall risk group; LLR: a locomotive impairment and low fall risk group; and LHR: a locomotive impairment and high fall risk group. " covariate. 


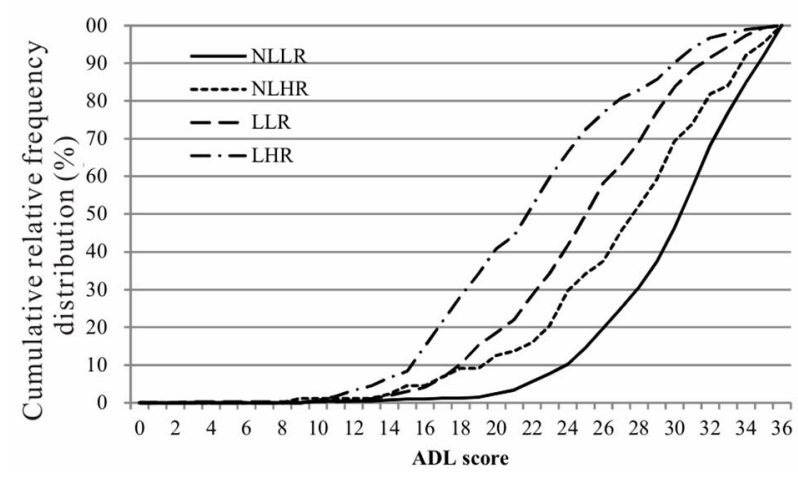

Figure 2. The cumulative relative frequency distribution curves for classification by locomotive organ impairment and potential for falling. NLLR: a no locomotive impairment and low fall risk group, NLHR: a no locomotive impairment and high fall risk group, LLR: a locomotive impairment and low fall risk group, and LHR: a locomotive impairment and high fall risk group.

lling elderly and establish appropriate measures for preventing falls. However, these approaches have not been sufficiently examined in the past.

Although there is no clear definition of a low-risk faller, it may be convenient to think of this group as having superior physical function, healthy locomotive organs, and low potential for falling. In this study, nearly $40 \%$ of the community-dwelling elderly had no locomotive impairment and a low potential for falling, and $14 \%$ of these reported a previous fall experience (low-risk faller). Therefore, a third of elderly individuals without problems in physical function and locomotive organs in this study had experienced falls, indicating that fallers include elderly individuals with varying fall risk factors.
In addition, nonfallers included elderly individuals with a high fall risk; nearly $17 \%$ of the elderly individuals in this study had both locomotive impairment and a high fall risk, and a third of participants (34\%) were high-risk nonfallers. Overlooking these high-risk nonfallers during risk assessment based on fall experience alone may be a serious problem. It is important to explore the characteristics of these target groups and determine appropriate fall prevention measures.

This study dealt with locomotive organ impairment, fall risk, and fall experience (within the last year) as important risk factors for future falls and examined the influence of these risk factors on physical function (ADL competence). In this study sample, fallers with locomotive impairment and a high fall risk exhibited the lowest ADL score, whereas fallers without locomotive impairment and with a low fall risk exhibited the highest score. Some inferences can be made from this finding.

First, there is a limitation to fall risk assessment based on fall experience alone. As shown in Figure 1, there were no clear differences in the distribution characteristics of ADL score between the faller and nonfaller groups, although there was a statistically significant difference in ADL score. A similar trend is exhibited in Figure 3. Categorizing all fallers as high-risk individuals may lead to the misinterpretation of low-risk individuals as high-risk individuals. In addition, there are elderly individuals with locomotive impairment and a high potential for falling but have not experienced previous falls. It is important to ensure that these high-risk nonfallers should not be evaluated as low-risk individuals.

Second, because locomotive organ health and fall risk are closely related to ADL competence, it is preferable to

Table 3. The results for classification by fall experience, locomotive organ impairment, and potential for falling.

\begin{tabular}{|c|c|c|c|c|c|c|c|c|c|}
\hline & \multirow{2}{*}{$\mathrm{n}$} & \multicolumn{4}{|c|}{ Mean age } & \multicolumn{4}{|c|}{ ADL score } \\
\hline & & Mean & SD & ANOVA & Multiple comparison & Mean & SD & ANCOVA & Multiple comparison \\
\hline $\begin{array}{l}\text { LHR-faller } \\
\text { (G1) }\end{array}$ & 118 & 76.1 & 7.1 & & & 21.8 & 5.7 & & \\
\hline $\begin{array}{l}\text { LHR-nonfaller } \\
\text { (G2) }\end{array}$ & 62 & 74.3 & 7.0 & & & 23.5 & 5.2 & & \\
\hline $\begin{array}{l}\text { LLR-faller } \\
\text { (G3) }\end{array}$ & 75 & 74.7 & 6.7 & & & 24.7 & 5.7 & & \\
\hline $\begin{array}{l}\text { LLR-nonfaller } \\
\text { (G4) }\end{array}$ & 298 & 73.6 & 7.0 & $F=11.9$ & G1 > G4, G5, G6, G7, G8/ & 25.6 & 5.0 & $F=38.3$ & $\begin{array}{c}\mathrm{G} 1, \mathrm{G} 2<\mathrm{G} 4, \mathrm{G} 5<\mathrm{G} 6, \mathrm{G} 8 / \\
\mathrm{G} 1<\mathrm{G} 2 /\end{array}$ \\
\hline $\begin{array}{l}\text { NLHR-faller } \\
\text { (G5) }\end{array}$ & 48 & 72.3 & 8.1 & $\mathrm{p}=0.00$ & G2, G3, G4 > G7, G8 & 27.0 & 6.1 & $\mathrm{p}=0.00$ & $\begin{array}{c}\mathrm{G} 3<\mathrm{G} 6<\mathrm{G} 8 / \mathrm{G} 3<\mathrm{G} 7 / \mathrm{G} 4 \\
<\mathrm{G} 6\end{array}$ \\
\hline $\begin{array}{l}\text { NLHR-nonfaller } \\
\text { (G6) }\end{array}$ & 40 & 70.8 & 6.4 & & & 28.0 & 5.1 & & \\
\hline $\begin{array}{l}\text { NLLR-nonfaller } \\
\text { (G7) }\end{array}$ & 359 & 70.7 & 6.5 & & & 29.9 & 4.4 & & \\
\hline $\begin{array}{l}\text { NLLR-faller } \\
\text { (G8) }\end{array}$ & 59 & 70.4 & 7.8 & & & 31.1 & 3.8 & & \\
\hline
\end{tabular}

NLLR-faller: nonfallers with no locomotive impairment and low fall risk; NLHR-nonfaller: nonfallers with no locomotive impairment and high fall risk, LLRnonfaller: nonfallers with locomotive impairment and low fall risk; LHR-nonfaller: nonfallers with locomotive impairment and high fall risk; NLLR-faller: fallers with no locomotive impairment and low fall risk; NLHR-faller: fallers with no locomotive impairment and high fall risk; LLR-faller: fallers with locomotive impairment and low fall risk; and LHR-faller: fallers with locomotive impairment and high fall risk. Statistical differences in mean ADL scores among these groups were confirmed by ANCOVA (adjusted by age effect). 


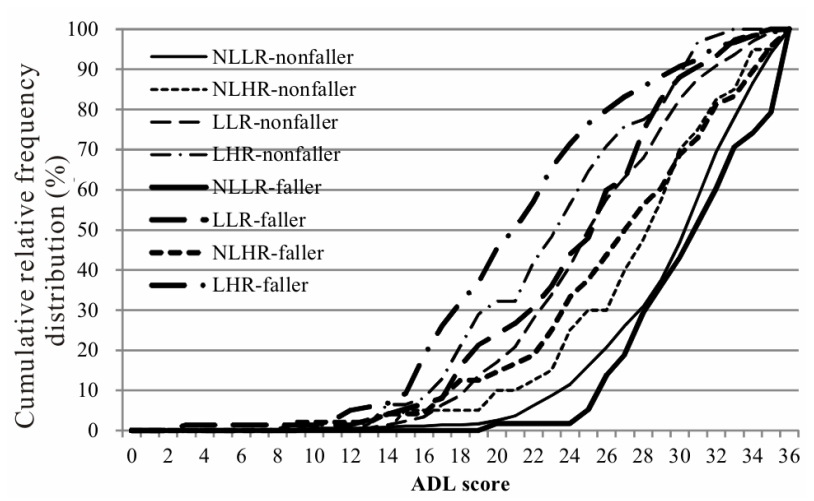

Figure 3. The cumulative relative frequency distribution curves classification by fall experience, locomotive organ impairment, and potential for falling. NLLR-faller: nonfallers with no locomotive impairment and low fall risk, NLHR-nonfaller: nonfallers with no locomotive impairment and high fall risk, LLR-nonfaller: nonfallers with locomotive impairment and low fall risk, LHR-nonfaller: nonfallers with locomotive impairment and high fall risk, NLLR-faller: fallers with no locomotive impairment and low fall risk, NLHR-faller: fallers with no locomotive impairment and high fall risk, LLRfaller: fallers with locomotive impairment and low fall risk, and LHR-faller: fallers with locomotive impairment and high fall risk. Statistical differences in mean ADL scores among these groups were confirmed by ANCOVA (adjusted by age effect).

assess a combination of these factors during fall risk assessments. As is obvious from the results of this study, there is a limitation to the effectiveness of risk stratification using fall experience alone. Grouping of individuals on the basis of a combination of locomotive organ health, fall risk level, and fall experience may provide the opportunity to screen a target group that requires primary preventive measures against falls. Understanding the fall risk profile together with the physical function characteristics is also helpful to determine adequate fall preventive measures.

Third, even the simple ADL check list used in this study can evaluate physical function as part of fall prevention screening of the community-dwelling elderly. Although physical function assessment based on a performance test may be desirable, there are difficulties in the application of performance tests because these include physical and personal costs as well as safety considerations. In contrast, a questionnaire-based assessment is a simple and versatile approach. The ADL assessment chart used in this study is also used to determine the suitability of a performance test (cutoff is 24 points). Among the eight groups classified by fall experience, locomotive organ impairment, and potential for falling in this study, the two groups with locomotive organ impairment and a high fall risk (LHR-nonfaller and LHR-faller) exhibited mean ADL scores that were less than 24 points, indicating the possibility that elderly individuals with locomotive impairment and a high fall risk cannot be easily subjected to performance-based physical function assessment.

\section{CONCLUSION}

This study classified a cohort of independent community-dwelling elderly individuals into several groups on the basis of key fall risk characteristics, namely fall experience, locomotive organ health, and potential for falling, and compared the physical function characteristics among groups. Classification of elderly individuals on the basis of these risk factors may help in the identification of target populations requiring primary preventive measures against falls, including high-risk fallers, low-risk fallers, and high-risk nonfallers; in addition, such a classification may be important in the assessment of fall risk characteristics among the community-dwelling elderly.

\section{ACKNOWLEDGEMENTS}

We are very grateful to the subjects for their enthusiastic participation during our measurements. The study was supported by the Japan Society for the Promotion of Science (Grant-in-Aid for Scientific Research (A) \#21240064). The authors would like to thank Enago (www.enago.jp) for the English language review.

\section{REFERENCES}

[1] Health, Labour and Welfare Ministry (2012) The 21st life tables, Complete life table.

www.mhlw.go.jp/english/database/db-hw/lifetb21th/index html

[2] World Health Organization (2011) World Health Statics 2010.

[3] American Geriatrics Society, British Geriatrics Society, and American Academy of Orthopaedic Surgeons Panel on Falls Prevention (2001) Guideline for the prevention of falls in older persons. Journal of the American Geriatrics Society, 49, 664-672. doi:10.1046/j.1532-5415.2001.49115.x

[4] Tinetti, M.E., Speechley, M. and Ginter, S.F. (1988) Risk factors for falls among elderly persons living in the community. The New England Journal of Medicine, 319, 17011707. doi:10.1056/NEJM198812293192604

[5] Nakamura, K. (2009) Locomotive syndrome: Disabilityfree life expectancy and locomotive organ health in a "super-aged” society. Journal of Orthopaedic Science, 14, 1-2. doi:10.1007/s00776-008-1302-y

[6] Gates, S., Smith, L.A., Fisher, J.D. and Lamb, S.E. (2008) Systematic review of accuracy of screening instruments for predicting fall risk among independently living older adults. Journal of Rehabilitation Research \& Development, 45, 1105-1116. doi:10.1682/JRRD.2008.04.0057

[7] Gregg, E.W., Pereira, M.A. and Caspersen, C.J. (2000) Physical activity, falls, and fractures among older adults: A review of the epidemiologic evidence. Journal of the American Geriatrics Society, 48, 883-893.

[8] Lord, S.R., Tiedemann, A., Chapman, G.K., et al. (2005) The effect of an individualized fall prevention program 
on fall risk and falls in older people: A randomized, controlled trial. Journal of the American Geriatrics Society, 53, 1296-1304. doi:10.1111/j.1532-5415.2005.53425.x

[9] Stalenhoef, P.A., Diederiks, J.P., Knottnerus, J.A., Kester, A.D. and Crebolder, H.F. (2002) A risk model for the prediction of recurrent falls in community-dwelling elderly: A prospective cohort study. The New England Journal of Medicine, 55, 1088-1094. doi:10.1016/S0895-4356(02)00502-4

[10] Nakamura, K. (2011) The concept and treatment of locomotive syndrome: Its acceptance and spread in Japan. Journal of Orthopaedic Science, 16, 489-491. doi:10.1007/s00776-011-0108-5

[11] Ministry of Health, Labour and Welfare (2010) Comprehensive survey of living conditions in 2010. http://www.mhlw.go.jp/toukei/saikin/hw/k-tyosa/k-tyosa1 $\underline{0 /}$

[12] Demura, S., Sato, S., Yokoya, T., et al. (2010) Examina- tion of useful items for the assessment of fall risk in the community-dwelling elderly Japanese population. Environmental Health and Preventive Medicine, 15, 169-179. doi:10.1007/s12199-009-0124-7

[13] Demura, S., Kasuga, K., Sato, S., et al. (2013) Determination of persons at a high risk of falling in a population of healthy community-dwelling elderly Japanese. International Journal of Gerontology, 7, 13-16. doi:10.1016/j.ijge.2012.05.007

[14] Sato, S., Demura, S., Shin, S., et al. (2012) Assessing a personal and population fall risk profile in Japanese community-dwelling elderly. Japan Journal of Test Evaluation and Physical Education Sports, 12, 49-55.

[15] Ministry of Education, Culture, Sports, Science and Technology (1999) Guideline for physical functional test. http://www.mext.go.jp/a_menu/sports/stamina/03040901. $\underline{\mathrm{htm}}$ 